Artigo Original

Original Article

\title{
Prevalência da Síndrome do Cólon Irritável em Pacientes com Fibromialgia
}

\section{Prevalence of Irritable Bowel Syndrome in Patients with Fibromyalgia}

\author{
Milton Helfenstein Jr. ${ }^{(1)}$, Roberto Heymann ${ }^{(2)}$, Daniel Feldman ${ }^{(3)}$
}

\section{RESUMO}

Introdução: a síndrome da fibromialgia (FM) é uma síndrome dolorosa crônica comum na população em geral. Não apresenta qualquer processo inflamatório, distrófico ou degenerativo, e é considerada como conseqüente a disfunções neurofisiológicas envolvendo, principalmente, o sistema nervoso central (SNC). A síndrome do cólon irritável (SCI) é considerada como uma entidade disfuncional visceral, caracterizada por fenômenos motores do trânsito gastrointestinal, possivelmente relacionados a alterações neurofisiológicas nas mesmas vias do SNC. Objetivo: estudar a prevalência da SCI em portadores de FM e verificar as suas características clínicas. Pacientes e Métodos: foram estudados 200 portadores da síndrome da FM. Resultados: verificou-se que, entre esses pacientes, 134 (67\%) apresentaram, concomitantemente, a SCI; entre esses, a maioria $(121=90,2 \%)$ sofria o subtipo constipativo da moléstia. Traço de personalidade e estado emocional associados à ansiedade e depressão foram evidenciados numa substancial proporção desses pacientes. Conclusão: entre os pacientes com FM, não houve diferenças clínicas entre aqueles com e sem a SCI.

Palavras-chave: fibromialgia, cólon irritável, dor.

\section{INTRODUÇÃO}

A FM é conceituada como sendo uma condição dolorosa crônica, caracterizada por dor difusa pelo corpo e pelo achado, no exame físico, de sensibilidade dolorosa aumentada em sítios anatômicos pré-determinados, conhecidos como tender points. Os critérios diagnósticos exigem a presença de dor difusa, afetando o esqueleto axial e periférico, acima e abaixo da linha da cintura, e o achado de pelo menos 11 de 18 tender points específicos. Estes critérios apresentam uma sensibilidade de $88,4 \%$ e uma

\begin{abstract}
Introduction: Fibromyalgia is a prevalent syndrome of widespread pain and multiple tender points, with no inflammatory, dystrophic or degenerative changes, probably related to abnormal central modulation of pain processing. Irritable bowel syndrome is considered a dysfunctional syndrome of visceral pain and/or alteration on gastrointestinal motility, also related to the same central nervous system (CNS) abnormalities. Objective: The aim of this study was to evaluate the prevalence of irritable bowel syndrome in patients with fibromyalgia and to establish their clinical characteristics. Patients and Methods:Two hundred consecutive patients with fibromyalgia syndrome were studied. Results: These, 134 (67\%) had irritable bowel syndrome. The most common presentation was the constipation subtype (121 $=90.2 \%)$. The majority of patients had high scores of anxiety and/ or depression. Conclusion: No differences were noted among patients with fibromyalgia without irritable bowel syndrome, and those with both conditions.
\end{abstract}

Keywords: fibromyalgia syndrome, irritable bowel syndrome, musculoskeletal pain.

especificidade de $81,1 \%$, demonstrando que o julgamento clínico é decisivo no diagnóstico ${ }^{(1,2)}$.

Apesar de esta síndrome estar clinicamente definida há muito tempo, sua etiopatogenia permanece obscura. As pesquisas atuais sustentam a hipótese de um distúrbio complexo da neuromodulação da dor, envolvendo principalmente o sistema nervoso central (SNC). Evidências de anormalidades centrais e neuro-hormonais têm sido declaradas há vários anos. Aceita-se um modelo de fisiopatologia que integra muitas das idéias publicadas e que sugere que o distúrbio primário na fibromialgia seria uma alteração

Disciplina de Reumatologia, Escola Paulista de Medicina, Universidade Federal de São Paulo (UNIFESP). Recebido em 14/04/05. Aprovado, após revisão, em 20/10/05

1. Assistente Doutor da Disciplina de Reumatologia da Universidade Federal de São Paulo (UNIFESP).

2. Assistente Doutor da Disciplina de Reumatologia da Universidade Federal de São Paulo (UNIFESP).

3. Professor Adjunto da Disciplina de Reumatologia da Universidade Federal de São Paulo (UNIFESP).

Endereço para correspondência: Milton Helfenstein, Rua Botucatu, 740, São Paulo, CEP 04023-900, SP, Brasil, telefone (11) 5579-6655, fax (11) 5093-7650 
em algum mecanismo central de controle da dor, o qual poderia resultar de uma disfunção de neurotransmissores. Tal disfunção neuro-hormonal incluiria uma deficiência de neurotransmissores inibitórios em níveis espinhais ou supraespinhais (serotonina, encefalina, norepinefrina e outros), ou uma hiperatividade de neurotransmissores excitatórios (substância P, glutamato, bradicinina e outros peptídeos), ou ainda ambas as condições poderiam estar presentes ${ }^{(2)}$. A deficiência de serotonina, um neuro-hormônio inibitório da dor, pode contribuir para anomalias do sono, depressão e amplificação da dor ${ }^{(4)}$. A liberação de substância $\mathrm{P}$, um neuro-hormônio excitatório, é influenciada pela deficiência de serotonina, seja no SNC, seja no sistema nervoso periférico e pode causar um aumento na percepção da dor. Alguns acreditam que o triptofano, um precursor da serotonina e também um neuromodulador, e outros nove aminoácidos, teriam sua concentração plasmática diminuída na FM e, ainda, que existiria uma maior afinidade na ligação da imipramina aos receptores serotoninérgicos plaquetários nesses pacientes $^{(5)}$. As pesquisas também evidenciaram diminuição dos níveis de triptofano e de outros aminoácidos, além de um aumento de concentração de substância $\mathrm{P}$, endorfinas e ácido 5-hidroxi-indolacético, no sangue e no líquor dos portadores de $\mathrm{FM}^{(6)}$.

A síndrome do cólon irritável (SCI) também corresponde a uma entidade nosológica de origem desconhecida, que não possui evidências de lesão tecidual. Tal síndrome é definida como uma combinação de sintomas gastrintestinais crônicos ou recorrentes, não explicados por anormalidades estruturais ou bioquímicas, a qual é atribuída ao intestino e associada a sintomas de dor e distúrbio da defecação e/ou sintomas de desconforto e distensão abdominal ${ }^{(7)}$. Apesar de sua origem ser desconhecida, a SCI é considerada como um distúrbio funcional, devido à ausência de uma lesão identificável. Diversos mecanismos fisiopatológicos foram hipotetizados e estudados, envolvendo o trânsito anormal do bolo fecal e dos gases, o papel do estresse, a hipersensitividade visceral, a mobilidade colônica alterada, entre outros. Esses mecanismos poderiam contribuir para os sintomas, de maneira isolada ou conjunta, além de um distúrbio cognitivo, podendo alterar o comportamento do paciente. Fatores genéticos, que podem afetar a sinalização da dor, e distúrbios do sistema nervoso central, que podem afetar o processamento de vias aferentes, têm sido postulados como fatores predisponentes, após algum tipo de exposição ambiental específica ${ }^{(8)}$. A ativação cerebral anormal pode ter um papel decisivo na SCI, pois o cérebro não é apenas importante para a interpretação de estímulos e a relação com memórias prévias, mas também exerce um efeito importante na sinalização ascendente da dor. O sistema antinociceptivo descendente, o qual inibe a transmissão da dor, também pode exercer um papel na síndrome e em outras síndromes dolorosas, como na $\mathrm{FM}^{(9,10)}$.

$\mathrm{Na}$ América do Norte, a SCI responde por 25 a $50 \%$ das consultas aos gastroenterologistas ${ }^{(11)}$, sendo que a prevalência desta síndrome na América do Norte é estimada entre 10 e $15 \%$ da população. Em relação ao sexo, a prevalência varia de uma predominância feminina 2:1 para uma proporção de 1:1. Entre os portadores, a prevalência é de aproximadamente $5 \%$, tanto no subtipo diarréico como no subtipo constipativo ${ }^{(12)}$. Um estudo constatou que a prevalência do subtipo constipativo foi de $5,2 \%$ e do subtipo diarréico de $5,5 \%$. Neste estudo, o sexo feminino predominou no subtipo constipativo $(6,7 \%$ no sexo feminino versus $3,5 \%$ no sexo masculino $)^{(13)}$.

Diversos critérios diagnósticos foram criados e modificados nas últimas décadas, entre eles Mainning (1978), Drossman (1979), Roma I (1989) e Roma II (1999). Os critérios atualmente utilizados são os de Roma $\mathrm{II}^{(14)}$ (Tabela 1).

TABela 1

Critérios de Mainning e Critérios de Roma II PARA A SÍNDROME DO CÓLON IRRITÁVEL (SCI)

\begin{tabular}{|c|c|}
\hline CRitério de Mainning (1978) & CRItério de Roma II (1999) \\
\hline $\begin{array}{l}\text { - Dor aliviada pela defecação } \\
\text { - Fezes amolecidas no início do } \\
\text { quadro doloroso } \\
\text { - Evacuação mais freqüente no } \\
\text { início do quadro doloroso } \\
\text { - Distensão abdominal visível } \\
\text { - Passagem de muco } \\
\text { - Sensação de esvaziamento } \\
\text { retal incompleto }\end{array}$ & $\begin{array}{l}\text { Dor ou desconforto por } 12 \\
\text { semanas nos últimos } 12 \text { meses, } \\
\text { associado a pelo menos } 2 \text { dos } 3 \\
\text { sintomas seguintes: } \\
1 \text { - Alívio com defecação } \\
2 \text { - Fezes amolecidas ou mais } \\
\text { freqüentes } \\
3 \text { - Fezes endurecidas ou menos } \\
\text { freqüentes } \\
\text { Sintomas cumulativos que } \\
\text { contribuem para o diagnóstico: } \\
\text { - Freqüência anormal de } \\
\text { evacuação (> } 3 \text { por dia ou < } 3 \\
\text { por semana) } \\
\text { - Formato anormal das fezes } \\
\text { (grumosa/endurecida ou } \\
\text { amolecida/aquosa) } \\
\text { - Passagem fecal anormal } \\
\text { (esforço, urgência ou sensação } \\
\text { de esvaziamento retal } \\
\text { incompleto) } \\
\text { - Passagem de muco } \\
\text { - Sensação de gases ou de } \\
\text { distensão abdominal }\end{array}$ \\
\hline
\end{tabular}


Os critérios de Roma II definiram e classificaram 24 "distúrbios gastrintestinais funcionais", termo utilizado para definir diversas situações de sintomas gastrintestinais, crônicos ou recorrentes, que não possuem uma fisiopatologia

TABELA 2

Critérios de Mainning e Critérios de Roma II PARA A SÍNDROME DO CÓlON IRRITÁVEL (SCI)

\begin{tabular}{|c|c|c|}
\hline $\begin{array}{l}\text { Constipação } \\
\text { funcional }\end{array}$ & $\begin{array}{l}\text { Dissinergia do } \\
\text { assoalho pélvico }\end{array}$ & $\begin{array}{c}\text { Subtipo } \\
\text { constipativo da SCI }\end{array}$ \\
\hline \multicolumn{3}{|c|}{$\begin{array}{l}\text { Sintomas contínuos ou recorrentes por pelo menos } 12 \text { semanas } \\
\text { nos últimos } 12 \text { meses; } 2 \text { ou mais sintomas de: }\end{array}$} \\
\hline $\begin{array}{l}\text { Esforço em menos de } \\
1 / 4 \text { das defecações }\end{array}$ & $\begin{array}{l}\text { Esforço em menos de } \\
1 / 4 \text { das defecações }\end{array}$ & $\begin{array}{l}\text { Dor abdominal } \\
\text { ou desconforto } \\
\text { aliviado(a) com } \\
\text { defecação ou } \\
\text { associada com } \\
\text { mudança na } \\
\text { freqüência ou } \\
\text { consistência das } \\
\text { fezes e um ou mais } \\
\text { sintomas de: }\end{array}$ \\
\hline $\begin{array}{l}\text { Fezes grumosas } \\
\text { e/ou endurecidas em } \\
\text { menos de } 1 / 4 \text { das } \\
\text { defecações }\end{array}$ & $\begin{array}{l}\text { Fezes grumosas } \\
\text { e/ou endurecidas em } \\
\text { menos de } 1 / 4 \text { das } \\
\text { defecações }\end{array}$ & $\begin{array}{l}\text { Esforço nas } \\
\text { defecações }\end{array}$ \\
\hline $\begin{array}{l}\text { Sensação de } \\
\text { evacuação } \\
\text { incompleta em } \\
\text { menos de } 1 / 4 \text { das } \\
\text { defecações }\end{array}$ & $\begin{array}{l}\text { Sensação de } \\
\text { evacuação } \\
\text { incompleta em } \\
\text { menos de } 1 / 4 \text { das } \\
\text { defecações }\end{array}$ & $\begin{array}{l}\text { Fezes grumosas e/ou } \\
\text { endurecidas }\end{array}$ \\
\hline $\begin{array}{l}\text { Menos que três } \\
\text { evacuações por } \\
\text { semana }\end{array}$ & $\begin{array}{l}\text { Evidência } \\
\text { manométrica, } \\
\text { eletromiográfica } \\
\text { ou radiográfica } \\
\text { de contração } \\
\text { inapropriada } \\
\text { ou insuficiência } \\
\text { de relaxamento } \\
\text { dos músculos do } \\
\text { assoalho pélvico } \\
\text { durante repetidas } \\
\text { tentativas de } \\
\text { defecação }\end{array}$ & $\begin{array}{l}\text { Sensação de } \\
\text { evacuação } \\
\text { incompleta }\end{array}$ \\
\hline $\begin{array}{l}\text { Sensação de } \\
\text { obstrução/bloqueio } \\
\text { ano-retal em } \\
\text { menos de } 1 / 4 \text { das } \\
\text { defecações }\end{array}$ & $\begin{array}{l}\text { Evidência de } \\
\text { forças propulsivas } \\
\text { adequadas durante } \\
\text { tentativas de } \\
\text { defecação }\end{array}$ & $\begin{array}{l}\text { Menos que três } \\
\text { defecações por } \\
\text { semana }\end{array}$ \\
\hline $\begin{array}{l}\text { Manobras manuais } \\
\text { para facilitar } \\
\text { menos de } 1 / 4 \text { das } \\
\text { defecações }\end{array}$ & $\begin{array}{l}\text { Evidência de } \\
\text { evacuação } \\
\text { incompleta }\end{array}$ & \\
\hline
\end{tabular}

Os sintomas em itálico não diferem nas três condições sindrômicas. claramente definida. Estes distúrbios foram divididos em seis subcategorias, baseadas em cinco regiões anatômicas: esofágica, gastrintestinal, colônica, biliar e ano-retal, juntamente com dor abdominal funcional (Tabela 2$)^{(15)}$. Separando esses distúrbios gastrintestinais funcionais em subgrupos, é possível identificar pacientes com um tipo de apresentação clínica homogênea. Uma publicação recente reconhece que os critérios de Roma II utilizados para distinguir o subtipo constipativo da SCI de uma constipação crônica funcional são tema de debate e de decisão arbitrária ${ }^{(16)}$. Cumpre esclarecer que constipação funcional é reconhecida como manifestação sintomática prolongada de defecação anormal, expressa por redução da freqüência de movimentos intestinais e/ou ato de evacuação alterado, em indivíduos que não possuem alterações orgânicas e que estão presentes com dois ou mais dos seguintes sintomas: 1) esforço para defecar; 2) fezes grumosas e/ou endurecidas; 3) sensação de evacuação incompleta e/ou 4) dois ou menos movimentos intestinais por semana ${ }^{(15)}$. Um estudo mostrou que a maior parte dos sintomas relevantes, citados por pacientes com constipação crônica foram: dor abdominal e/ou distensão, flatulência e dor de cabeça ${ }^{(17)}$. Desta maneira, a constipação funcional se apresenta clinicamente como uma síndrome, cujo aspecto central é representado por dois ou mais dos critérios diagnósticos de Roma II acompanhados por nenhum, um ou mais sintomas (Tabela 2). Estudar bem como fazer a tabela abaixo. Atentar para o conteúdo e não seguir as colunas, senão ficará sem sentido.

A classificação de Roma II dividiu em três categorias funcionais os distúrbios apresentados com sintomas intestinais de constipação: SCI predominantemente constipativa, constipação funcional e dissinergia do assoalho pélvico. Entretanto, pode ser difícil, ou até mesmo impossível, diferenciar estas três condições, porque dividem sintomas intestinais idênticos, que podem estar presentes nos indivíduos que também se queixam de desconforto ou dor abdominal ${ }^{(18)}$.

A prevalência da constipação funcional varia com as diferentes definições utilizadas e com as populações investigadas. Utilizando o critério de Roma II, varia entre 3 e 16,7\%(15,19). Sintomas relacionados à constipação foram reportados em $4,6 \%$ da população americana em um vasto estudo epidemiológico $^{(20)}$.

Curiosamente, 32 a $65 \%$ dos pacientes com SCI, também preenchem critérios para a $\mathrm{FM}^{(21,22)}$. É interessante notar que alguns estudos sugerem que o sexo dos pacientes é altamente associado com a concomitância dessas condições. Por exemplo, em um estudo, a SCI foi mais comum entre mulheres (40\%) do que em homens (14\%) com FM. ${ }^{(23)}$ 


\section{OBJETIVO}

Este estudo teve como objetivo avaliar a prevalência da SCI em pacientes com FM e caracterizar o respectivo quadro clínico desses indivíduos.

\section{PACIENTES E MÉTODOS}

Foram estudados 200 pacientes com diagnóstico de FM, acompanhados no ambulatório da disciplina de reumatologia da Escola Paulista de Medicina (UNIFESP) e que preenchiam os critérios para a classificação e diagnóstico da FM, elaborados pelo Colégio Americano de Reumato$\operatorname{logia}{ }^{(1)}$.

O critério de seleção dos pacientes foi o de chegada ao respectivo serviço de maneira consecutiva. Todos deviam ter mais do que 18 anos de idade. Os pacientes não poderiam estar em uso de opióides ou de medicamentos obstipantes. Pacientes portadores de hipotireoidismo ou de conhecida parasitose intestinal foram excluídos do estudo.

Todos os pacientes foram avaliados por um único investigador, seguindo um protocolo clínico que consistia em:

1. Dados demográficos, incluindo dados ocupacionais, tipo de trabalho, lazer e esporte;

2. História clínica do quadro doloroso e do quadro intestinal;

3. Critérios diagnósticos para a SCI e para o subtipo constipativo $^{(14)}$;

4. Questionários específicos para avaliação de:

a. Impacto da doença sobre a qualidade de vida: através do Fibromyalgia Impact Questionnaire (FIQ) ${ }^{(24,25)}$;

b. Status psicológico: através da utilização do Inventário de Ansiedade Traço-Estado (IDATE), desenvolvido por Spielberger et $a^{(26)}$ e posteriormente traduzido e validado para o Brasil por Biaggio e Natalício ${ }^{(27)}$, e da utilização do Inventário Beck de depressão, desenvolvido por Beck et $a^{(28)}$, traduzido por V Ribeiro ${ }^{(29)}$ e posteriormente validado para o Brasil ${ }^{(30,31)}$.

5. Exame físico geral e do aparelho locomotor.

6. Exame dos tender points: os 18 tender points estabelecidos pelo Colégio Americano de Reumatologia ${ }^{(1)}$ foram pesquisados através de digitopressão ${ }^{(32-34)}$; foram também pesquisados dez pontos-controle: antebraços, dorso das mãos, unha dos polegares, dorso dos pés, fronte e mento.

7. Exames complementares realizados quando necessário: hemograma completo (metodologia automatizada), velocidade de hemossedimentação (Wintrobe ou Westergren), creatina-fosfoquinase (método cinético), fator antinuclear (Hep-2), fator reumatóide (látex), proteína C- reativa (nefelometria), triiodotironina, tetraiodotironina, hormônio tireoestimulante (ensaio imunofluorométrico) e alfa-1 glicoproteína ácida (imunonefelometria). Quando necessário, outros exames foram realizados, como radiografia (raio X), ultra-sonografia (US), eletroneuromiografia (EMG), tomografia computadorizada (TC) e ressonância magnética $(\mathrm{RM})$.

\section{RESULTADOS}

A quase totalidade dos portadores da FM pertenceu ao sexo feminino (199). A idade média era de 57 anos. Entre os 200 pacientes estudados, 134 (67\%) preencheram critérios para a SCI (Tabela 1).

TABELA 3

Pacientes com Fibromialgia $(\mathrm{N}=200)$

\begin{tabular}{cc} 
Sexo & $199(99,5 \%)$ feminino; $1(0,5 \%)$ masculino \\
Idade & 57,1 anos (entre 20 e 77 anos) \\
SCI & $134(67 \%)$ \\
\hline
\end{tabular}

Entre os 134 pacientes com SCI, 121 (90,2\%) apresentavam padrão constipativo, 5 (3,8\%) padrão diarréico, $3(2,2 \%)$ padrão de dor abdominal com fezes amolecidas e $5(3,8 \%)$ pacientes tinham hábitos alternantes de diarréia e constipação. Todos pertenciam ao sexo feminino (Tabela 2).

TABELA 4

Pacientes com Fibromialgia e Ś́ndrome do Cólon IRRITÁVEL (N=134)

\begin{tabular}{lc}
\hline PADRÃo & $\mathbf{n}(\%)$ \\
Constipativo & $121(90,2 \%)$ \\
\hline Diarréico & $5(3,8 \%)$ \\
Dor Abdominal & $3(2,2 \%)$ \\
\hline Alternante (Diarréia e Constipação) & $5(3,8 \%)$ \\
\hline
\end{tabular}

Não houve diferença de apresentação clínica entre os pacientes com FM sem SCI e os pacientes com FM e SCI (Tabela 3).

$\mathrm{Na}$ avaliação da qualidade de vida, através do FIQ, foi verificado que a maioria dos pacientes percebe a dor como sendo de forte intensidade, o cansaço como sendo relevante, assim como sentem dificuldades para executar tarefas da rotina diária. No entanto, foi constatado que a capacidade funcional foi pouco alterada (Tabela 4).

Nota-se que os pacientes sentem a dor como de forte intensidade. Os achados foram similares entre os grupos de pacientes com FM, com e sem SCI.

Nos inventários utilizados para avaliar o traço e estado 
de ansiedade e a depressão, observou-se que a maioria dos pacientes que apresentavam concomitantemente FM e SCI exibiram um traço de personalidade associado à ansiedade. A maioria também apresentou estado de ansiedade e depressão significativo (Tabela 5 ).

Nota-se que em todos os parâmetros avaliados não houve diferença estatística entre os dois grupos.

\section{TABELA 5}

CARACTERÍSTICAS CLÍNICAS DOS PACIENTES COM FM COM E SEM SCI

\begin{tabular}{|c|c|c|}
\hline SINTOMA & $\begin{array}{l}\text { SF com SCI } \\
(n=134)\end{array}$ & $\begin{array}{l}\text { SF sem SCI } \\
\quad(n=66)\end{array}$ \\
\hline Rigidez & $133(99 \%)$ & $65(99 \%)$ \\
\hline Distúrbio do sono & $130(97 \%)$ & $63(95 \%)$ \\
\hline Fadiga & $126(94 \%)$ & $61(92 \%)$ \\
\hline Parestesia & $114(85 \%)$ & $56(85 \%)$ \\
\hline Dificuldade de memória & $105(78 \%)$ & $51(77 \%)$ \\
\hline Palpitação & $99(74 \%)$ & $48(73 \%)$ \\
\hline Tontura & $98(73 \%)$ & $47(71 \%)$ \\
\hline Sensação de inchaço & $94(70 \%)$ & $46(70 \%)$ \\
\hline Dor torácica & $94(70 \%)$ & $45(68 \%)$ \\
\hline Síndrome seca & $90(67 \%)$ & $44(67 \%)$ \\
\hline Dificuldade de concentração & $88(66 \%)$ & $44(67 \%)$ \\
\hline Zumbido & $76(57 \%)$ & $37(56 \%)$ \\
\hline Epigastralgia & $71(53 \%)$ & $35(53 \%)$ \\
\hline Dispnéia & $70(52 \%)$ & $34(52 \%)$ \\
\hline Enjôo & $67(50 \%)$ & $32(48 \%)$ \\
\hline
\end{tabular}

* FM = fibromialgia; SCI= síndrome do cólon irritável

TABela 6

Fibromyalgia Impact Questionnatre (FiQ) nos Pacientes COM FM E COM SCI

\begin{tabular}{|c|c|c|c|}
\hline$S F+S^{*} I^{*}(n=134)$ & MÉDIA & $\begin{array}{l}\text { DESVIO } \\
\text { PADRÃO }\end{array}$ & MÍN - MÁX \\
\hline FIQ Total & 7,68 & 0,94 & $3,68-9,53$ \\
\hline $\mathrm{FIQ}_{1-10}$ (capacidade funcional) & 1,26 & 2,18 & $0-9,66$ \\
\hline $\mathrm{FIO}_{11}$ (sem sintomas) & 9,66 & 1,13 & $2,86-10$ \\
\hline $\mathrm{FIO}_{12^{*}}$ (faltas no trabalho) & - & - & - \\
\hline $\mathrm{FIQ}_{13}$ (dificuldade para tarefas) & 8,16 & 1,54 & $3-10$ \\
\hline $\mathrm{FIQ}_{14}$ (escala de dor) & 9,61 & 0,92 & $5-10$ \\
\hline $\mathrm{FIQ}_{15}$ (cansaço) & 8,01 & 1,78 & $3-10$ \\
\hline $\mathrm{FIQ}_{16}$ (despertar) & 7,54 & 1,89 & $1-10$ \\
\hline $\mathrm{FIQ}_{17}$ (rigidez) & 7,95 & 1,57 & $2-10$ \\
\hline $\mathrm{FIO}_{18}$ (ansiedade) & 8,42 & 2,05 & $0-10$ \\
\hline $\mathrm{FIQ}_{19}$ (depressão) & 7,98 & 2,41 & $0-10$ \\
\hline
\end{tabular}

* FM = fibromialgia: SCI= síndrome do cólon irritável
TABELA 7

Traço e Estado de Ansiedade e Depressão nos Pacientes COM FM COM E SEM SCI

\begin{tabular}{lccc}
\hline ANSIEDADE - TRAÇO & $\begin{array}{c}\text { FM com SCI } \\
(\mathbf{n = 1 3 4 )}\end{array}$ & $\begin{array}{c}\text { FM sem SCI } \\
(\mathbf{n}=66)\end{array}$ & $\mathbf{p}$ \\
Normal & $11(8 \%)$ & $5(8 \%)$ & NS \\
Média & $29(22 \%)$ & $15(23 \%)$ & NS \\
Alta & $94(70 \%)$ & $46(70 \%)$ & NS
\end{tabular}

ANSIEDADE - ESTADO

$\begin{array}{lccc}\text { Normal } & 3(2 \%) & 1(2 \%) & \text { NS } \\ \text { Média } & 13(10 \%) & 7(11 \%) & \text { NS } \\ \text { Alta } & 117(87 \%) & 59(89 \%) & \text { NS }\end{array}$

\section{DEPRESSÃO}

$\begin{array}{lccc}\text { Normal } & 12(9 \%) & 6(9 \%) & \text { NS } \\ \text { Leve } & 36(27 \%) & 18(27 \%) & \text { NS } \\ \text { Moderada } & 45(34 \%) & 23(35 \%) & \text { NS } \\ \text { Grave } & 41(31 \%) & 19(29 \%) & \text { NS }\end{array}$

* FM = fibromialgia; SCI= síndrome do cólon irritável

\section{DISCUSSÃO}

Entre os 200 pacientes com FM estudados, 134 (67\%) apresentavam SCI. Este dado é semelhante aos dados encontrados na literatura, os quais relatam que entre 41 e $60 \%$ dos pacientes apresentam essa disfunção intestinal ${ }^{(35-39)}$. O padrão de SCI que predominou foi o constipativo (90,2\%). Nem todos os pacientes se recordavam se a constipação antecedeu o surgimento de dor musculoesquelética difusa. No entanto, uma pequena proporção dos pacientes $(22 \%)$ afirmou que o quadro constipativo teve surgimento antes da dor difusa.

Este é o primeiro trabalho na literatura que aponta para o mais freqüente subtipo de SCI que se associa à FM. Este também é o primeiro estudo que compara as características clínicas de pacientes com FM, que apresentam concomitantemente SCI, com os portadores de FM sem a presença desta disfunção intestinal. Não houve diferença quanto às manifestações clínicas entre esses dois grupos de pacientes.

Esclarece-se que não foram realizados exames parasitológicos de fezes nos pacientes; de qualquer forma, cumpre lembrar que apenas 3,8\% apresentou o padrão diarréico. Assim sendo, torna-se difícil acreditar que uma parasitose intestinal tenha influenciado os resultados desse estudo.

A capacidade funcional, avaliada através das 19 questões do FIQ, demonstrou índices elevados e significativos. Chamam a atenção os baixos índices obtidos no $\mathrm{FIQ}_{1-10} \mathrm{e}$ os 
altos índices dos outros parâmetros avaliados. Os dez itens do $\mathrm{FIQ}_{1-10}$ compõem uma escala física funcional, na qual cada item é graduado de acordo com a freqüência com que cada indivíduo executa as funções questionadas. Constatouse que a maioria dos indivíduos encontra-se apta a realizar suas tarefas de rotina diária. Os outros nove domínios do $\mathrm{FIQ}_{11-19}$ envolvem questões subjetivas e a grande maioria dos pacientes referiu um forte impacto nesse sentido. Estas questões se referem ao número de dias que se sentiam bem na semana da avaliação clínica, à dificuldade em trabalhar (a maioria no próprio ambiente doméstico), à intensidade da dor, à fadiga, à sensação ao despertar, à rigidez, à ansiedade e à depressão. Tais questões são conhecidamente influenciadas por fatores emocionais e sociais. Os resultados do FIQ demonstram que a enfermidade causa um importante impacto na vida desses pacientes. $\mathrm{O} \mathrm{FIQ}_{12}$ não teve aplicabilidade, porque a grande maioria estava envolvida em trabalho doméstico (donas de casa).

Foram freqüentes os índices elevados de ansiedade e os índices de depressão moderada e grave, sugerindo que estas síndromes disfuncionais abrangem manifestações que resultam não apenas de interações físicas, mas também de interações psicológicas. A abordagem da maioria desses pacientes deve incluir uma avaliação psicológica. Ressalta-se que os aspectos psicológicos mostraram-se não ser os únicos fatores etiológicos e/ou agravantes destas síndromes.

Esses resultados indicam uma forte relação entre essas duas entidades nosológicas, sugerindo que esses pacientes possam, talvez, apresentar o mesmo espectro de uma única síndrome disfuncional, provavelmente relacionada a distúrbios em segmentos possíveis dos mecanismos de transmissão da dor, tanto em sistema nervoso periférico como em SNC, com atenção principal à serotonina. Esta, que é um neuro-hormônio inibitório da dor, exerce um papel primordial, tanto na percepção da dor como também na alteração da motilidade intestinal, pois está relacionada com a movimentação do intestino e com a secreção da água e de eletrólitos. Atualmente, é sabido que a serotonina está presente na mucosa do tubo digestivo, sendo sintetizada pelas células enterocromoafins do intestino. A sua eliminação ocorre no próprio intestino, através de um sistema similar ao do SNC, que é executado pelo próprio enterócito. Dentro do tubo digestivo, os receptores mais importantes para a serotonina são os do tipo 1,3 e 4 . O receptor do tipo 1 está relacionado ao relaxamento da fibra muscular intestinal e os outros estão relacionados à contração pró-cinética do tubo (estimulação e movimentação peristáltica colônica).

Diversas influências psicossociais estão significativamente envolvidas nas manifestações destas duas entidades.

Devido ao alto grau de superposição, uma triagem para condições mórbidas associadas deve ser sempre feita na avaliação clínica de pacientes com suspeita de FM.

Um melhor entendimento dos mecanismos fisiopatológicos, envolvidos nesses dois distúrbios funcionais - FM e SCI - particularmente dos fenômenos psicossociais, dos distúrbios motores e das sensações viscerais, proporcionarão avanços na terapia medicamentosa, objetivando uma melhor abordagem destas duas entidades nosológicas.

Nos últimos anos, atenção tem sido dada à investigação das estruturas do SNC que estão envolvidas na percepção da dor, e também na estimulação colônica. Estudos de imagem funcional na SCI, envolvendo tomografia por emissão de fóton único (SPECT) e RM funcional, demonstraram um aumento significativo no fluxo sanguíneo em área cortical durante antecipação e estimulação colônica ${ }^{(40,41)}$. Estímulos dolorosos viscerais e somáticos estão fortemente relacionados com áreas distintas na região cortical anterior ${ }^{(42)}$. Outros estudos, também utilizando SPECT, no sentido de estudar fluxo sanguíneo cerebral em pacientes com FM, demonstraram diminuição significativa do fluxo sanguíneo nas regiões bilateral do tálamo e do núcleo caudado cerebral desses pacientes, quando comparados com indivíduos saudáveis ${ }^{(43)}$. Esses achados trazem implicações importantes, uma vez que áreas cerebrais exercem um papel primordial na integração de sinais da dor e nas gerações de sinais que regulam o eixo hipotálamo-hipófise-adrenal e a motilidade gastrintestinal. Assim sendo, possivelmente, alterações neurofuncionais e do fluxo sanguíneo cerebral podem estar envolvidas na fisiopatologia dessas duas síndromes.

\section{CONCLUSÃO}

Entre os 200 pacientes estudados com FM, 134 (67\%) também sofriam de SCI. Entre esses portadores de SCI, 92\% possuíam o tipo constipativo da síndrome. Distúrbios psicológicos, envolvendo ansiedade e depressão, foram constatados numa proporção significativa desses pacientes, no entanto, não houve diferenças clínicas entre os portadores de FM com e sem a SCI quanto aos aspectos psicológicos. Os portadores de FM e SCI demonstraram que essas moléstias causam um importante impacto na rotina diária, conforme constatado nos diversos parâmetros avaliados através do FIQ. No entanto, todos possuem capacidade para efetuar as suas tarefas, mesmo que demorem um tempo maior para a execução das mesmas. Apesar de sentirem muita dificuldade não estão incapacitados funcionalmente. 
O reconhecimento desta superposição de sintomas nesses pacientes permite clarificar diversos estados de dor musculoesquelética crônica e de distúrbios gastrintestinais, que têm sido erroneamente interpretados como outras

\section{REFERÊNCIAS}

1. Wolfe F, Smythe HA, Yunus MB et al: The American College of Rheumatology 1990. Criteria for the classification of fibromyalgia: report of the multicentre criteria committee. Arthritis Rheum 33: 160-172,1990.

2. Wolfe $\mathrm{F}$ and The Vancouver Fibromyalgia Consensus Group: The fibromyalgia syndrome: a consensus report on fibromyalgia and disability. J Rheumatol 23: 534-539, 1996.

3. Yunus MB: Towards a model of pathophysiology of fibromyalgia: aberrant central pain mechanisms with peripheral modulation. J Rheumatol 19: 846-849, 1992.

4. Russell IJ. Neurohormonal aspects of fibromyalgia syndrome - Rheum Dis Clin N Am 15: 149-168, 1989.

5. Russell IJ, Vaeroy HJ, Javors M et al: Cerebrospinal fluid biogenic amines in fibromyalgia/fibrositis syndrome and rheumatoid arthritis. Arthritis Rheum 35: 550-556, 1992.

6. Yunus MB, Dailey JW, Aldag JC et al: Plasma tryptophan and other amino acids in primary fibromyalgia: a controlled study. J Rheumatol 19: 90-94, 1992.

7. American Gastroenterological Association: American Gastroenterological Association position statement: irritable bowel syndrome. Gastroenterology 112: 2118-2119, 1997.

8. Talley NJ, Spiller R: Irritable bowel syndrome: a little understood organic bowel. Lancet 360: 555-564, 2002.

9. Bradley LA, McKendree-Smith NL, Alberts KR et al: Use of neuroimaging to understand abnormal pain sensitivity in fibromyalgia. Curr Rheumatol Rep 2: 141-148, 2000.

10. Naliboff BD, Derbyshire SW, Munakata J et al: Cerebral activation in patients with irritable bowel syndrome and control subjects during rectosigmoid stimulation. Psychosom Med 63: 365-375, 2001.

11. Mitchell CM, Drossman DA: Survey of the AGA membership relating to patients with functional gastrointestinal disorders. Gastroenterology 92: 1282-1284, 1987.

12. Saito YA; Schoenfeld P; Locke GR III: The epidemiology of irritable bowel syndrome in North America: A systematic review. Am J Gastroenterol 97: 1910-1915, 2002.

13. Talley NJ, Zinsmeister AR, Melton LJ: Irritable bowel syndrome in a community: symptom subgroups, risk factors, and healthcare utilization. Am J Epidemiol 142: 76-83, 1995.

14. Thompson WG, Longstreth GF, Drossman DA et al: Functional bowel disorders and functional abdominal pain. Gut 45: 11431147,1999 .

15. Drossman DA, Corazziari E, Talley NJ et al (eds): Rome II Functional Gastrointestinal Disorders. McLean, VA: Degnon Associates, 2000.

16. Talley NJ: Differentiating functional constipation from constipation-predominant irritable bowel syndrome: management implications. Rev Gastroenterol Disord 5:1-9, 2005. moléstias e, conseqüentemente, poupará tempo e gastos com investigações diagnósticas desnecessárias e, ainda, proporcionará a elaboração de estratégias terapêuticas mais eficientes, melhorando o prognóstico desses pacientes.

17. Corazziari E: Definition and epidemiology of functional gastrointestinal disorders. Best Pract Res Clin Gastroenterol 18: 613-631, 2004.

18. Corazziari E, Badiali D: How many chronic functional constipations? http://www.anemgi.org/htlm/introduction/ html. Último acesso em 17 de março de 2005.

19. Pare P, Ferrazzi S, Thompson WG et al: An epidemiological survey of constipation in Canada: definitions, rates demographics, and predictors of health care seeking. Am J Gastroenterol 96: 3130-3137, 2001.

20. Stewart WF, Liberman FJN, Sandler RS et al: Epidemiology of constipation (epoc) study in the United States: relation of clinical subtypes to sociodemographic features. Am J Gastroenterol 94: 3530-3540,1999.

21. Veale D, Kavanagh G, Fielding JF, Fitzgerald O: Primary fibromyalgia and the irritable bowel syndrome: different expressions of a common pathogenetic process. Brit J Rheumatol 30: 220-222,1991.

22. Sperber AD, Atzmon Y, Neumann L et al: Fibromyalgia in the irritable bowel syndrome: studies of prevalence and clinical implications. Am J Gastroenterol 94: 3541-3546,1999.

23. Yunus MB, Inanici F, Aldag JC, Mangold RF: Fibromyalgia in men: comparison of clinical features with women. J Rheumatol 27: 485-490, 2000.

24. Burckhardt CS, Clark SR, Bennett RM: The fibromyalgia impact questionnaire: development and validation. J Rheumatol 18 : 728-733, 1991 .

25. Bennett RM, Burckhardt CS, Clark SR: Fibrositis impact questionnaire (FIQ) users guide. Division of Arthritis \& Rheumatic Diseases. Oregon Health Sciences University. Portland, Oregon, 1991.

26. Spielberger CD, Gorsuch RL, Lushene RE: Manual for the state - trait anxiety inventory. California: Consulting Psychologist Press, 1970.

27. Biaggio AMB, Natalicio L: Inventário de ansiedade traço-estado (IDATE). Rio de Janeiro: Centro Editor de Psicologia Aplicada (CEPA), 1971.

28. Beck AT, Ward $\mathrm{CH}$, Mendelson $\mathrm{M}$ et al: An inventory for measuring depression. Arch Gen Psychiatry 4: 53-63,1961.

29. Beck AT, Rush AJ, Shaw BF e cols: Terapia cognitiva da depressão (traduzido por Vera Ribeiro). Zahar, Rio de Janeiro, 1982.

30. Gorenstein C, Andrade L, Pompeia S: Scores of Brazilian university students on the beck depression and the state-trait anxiety inventories. Psychological Reports 77: 635-641,1995.

31. Gorenstein C, Andrade L: Validation of a Portuguese version of the Beck depression inventory and the state-trait anxiety inventory in Brazilian subjects. Braz J Med Biol Res 29: 453457, 1996.

32. Cott A, Parkinson W, Bell MJ et al: Interrater reliability of the tender point criterion for fibromyalgia. J Rheumatol 19: 1955- 
1959, 1992.

33. Tunks E, Mc Cain GA, Hart LE et al: The reliability of examination for tenderness in patients with myofascial pain, chronic fibromyalgia and controls. J Rheumatol 22: 944-952, 1995.

34. Jacobs JW, Geenen K, Van der Heide A et al: Are tender points scores assessed by manual palpation in fibromyalgia reliable? An investigation into the variance of tender point scores. Scand J Rheumatol 24: 243-247, 1995.

35. Goldenberg DL: Fibromyalgia syndrome: an emerging but controversial condition. JAMA 257: 2782-2787, 1987.

36. Romano TJ: Coexistence of irritable bowel syndrome and fibromyalgia. West Virg Med J 84: 16-18, 1988.

37. Yunus MB, Masi AT, Aldag JC: A controlled study of primary fibromyalgia syndrome: clinical features and association with other functional syndromes. J Rheumatol 16: 62-71, 1989.

38. Triadafilopoulos G, Simms RW, Goldenberg DL: Bowel dysfunction in fibromyalgia syndrome. Digest Dis Sci 36: 59-
64,1991 .

39. Nishikai M: Fibromyalgia in Japanese. J Rheumatol 19: 110114, 1992.

40. Silverman DH, Munakata JA, Ennes H, Mandelkern MA, Hoh CK, Mayer EA: Regional cerebral activity in normal and pathological perception of visceral pain. Gastroenterology 112: 64-72, 1997.

41. Mertz H, Morgan V, Tanner G et al: Regional cerebral activation in irritable bowel syndrome and control subjects with painful and nonpainful rectal distention. Gastroenterology 118: 842848, 2000.

42. Rainville P, Duncan GH, Price DD, Carreir B, Bushnell MC: Pain affect encoded in human anterior cingulate but not somatosensory cortex. Science 1997 277: 968-971, 1997.

43. Mountz JM, Bradley LA, Modell JG et al: Fibromyalgia in women. Arthritis Rheum 38: 926-938, 1995. 